\title{
Preface to the Special Issue on "Recent Progress in Modeling, Data-processing and Control of Ironmaking Process"
}

\author{
Tatsuro ARIYAMA \\ Institute of Multidisciplinary Research for Advanced Materials, Tohoku University, 2-1-1 Katahira, Aoba-ku, Sendai $980-8577$ \\ Japan.
}

Recently, due to fear of the global warming, and the stable and high productivity derived from a strong demand of the steel market, the requirements on the ironmaking process have shown a remarkable change. For example, looking back over the last decades in the steel industry, we have focused on the increase in steel production and utilization of various resources to decrease the steel production cost. Today, however, low reducing agent operation is being actively pursued due to the global warming issue. Large blast furnaces with inner volumes exceeding $5000 \mathrm{~m}^{3}$ are constructed in some countries such as China and Japan, and accurate and reliable control of their operation is required to attain the above target. To achieve this, mathematical models of the ironmaking process can play an important role. Various mathematical models of the blast furnace based on advanced methods such as the discrete element method have recently been developed. Alternatively, datadriven modeling is also being broadly investigated and today exhibits great potential for describing the complex behavior of ironmaking processes. These new methods are expected to be able to further improve model accuracy and also to shed more light on how to optimize the blast furnace process operation in the future. Since these requirements are common to every country in the world, this special issue on "Recent Progress in Modeling, Data-processing and Control of Ironmaking" was planned by the following editors under the support of the ISIJ. The purpose of this special issue is to present new developments in the modeling, data-processing and control of the ironmaking process. Novel findings and useful informations on the ironmaking process are included in this special issue. We sincerely hope that this issue will contribute to the progress of ironmaking research through exchange and sharing of enlightening ideas.

Editors

Prof. Henrik Saxén

Thermal and Flow Engineering Laboratory

Abo Akademi University, Finland

Prof. Chuanhou Gao

Department of Mathematics

Zhejiang University, China

Prof. Tatsuro Ariyama

Institute of Multidisplinary Research for Advanced Materials

Tohoku University, Japan

Dr. Takanobu Inada

Ironmaking Research \& Development Department

Corporate Research \& Development Laboratories

Sumitomo Metal Industries, Ltd., Japan 